Relations industrielles

Industrial Relations

\title{
A Primer on American Labor Law, by W.B. Gould, Cambridge, Mass., The MIT Press, 1982, 242 pp., ISBN 0-262-070-87-1.
}

\section{Pierre Verge}

Volume 38, numéro 1, 1983

URI : https://id.erudit.org/iderudit/029338ar

DOI : https://doi.org/10.7202/029338ar

Aller au sommaire du numéro

Éditeur(s)

Département des relations industrielles de l'Université Laval

ISSN

0034-379X (imprimé)

1703-8138 (numérique)

Découvrir la revue

Citer ce compte rendu

Verge, P. (1983). Compte rendu de [A Primer on American Labor Law, by W.B.

Gould, Cambridge, Mass., The MIT Press, 1982, 242 pp., ISBN 0-262-070-87-1.]

Relations industrielles / Industrial Relations, 38(1), 180-181.

https://doi.org/10.7202/029338ar

Tous droits réservés @ C Département des relations industrielles de l'Université Laval, 1983
Ce document est protégé par la loi sur le droit d'auteur. L’utilisation des services d'Érudit (y compris la reproduction) est assujettie à sa politique d'utilisation que vous pouvez consulter en ligne.

https://apropos.erudit.org/fr/usagers/politique-dutilisation/ 
information, to be consulted frequently on its various facts and themes. In other words, the author has provided us with a veritable encyclopedia regarding the first century of Canada's labour history, which no serious labour specialist can afford to neglect.

James THWAITES

Université Laval

A Primer on American Labor Law, by W.B. Gould, Cambridge, Mass., The MIT Press, 1982, 242 pp., ISBN 0-262-070 87-1.

Voilà un ouvrage relativement bref dont la parution comble une lacune aussi étonnante que manifeste: l'absence relative de synthèses véritables du droit du travail américain, vaste domaine quasi monopolisé par des études dont la technicité rend plutôt difficile au lecteur - à tout le moins le lecteur étranger - d'appréhender les données essentielles du système en cause. Le professeur Gould, de Stanford University, réalise au surplus son entreprise en se permettant à l'occasion des rapprochements particuliers avec quelques systèmes étrangers - notamment ceux d'Angleterre, d'Allemagne, de Suède et du Japon -, ce qui est également peu fréquent dans les présentations connues du sujet.

La quasi-totalité des douze chapitres sont consacrés au droit de la négociation collective, ce qui reproduit assez fidèlement l'atrophie systémique des rapports collectifs du travail. Cet ensemble central est lui-même précédé d'une brève mise en contexte en fonction essentiellement des structures et des attitudes syndicales américaines et, surtout, d'une substantielle fresque de la période préWagner Act: histoire des tentatives répétées du travail organisé d'utiliser sa force politique pour contrer des attitudes jurisprudentielles défavorables et dont le dernier succès sera l'adoption du Norris-La Guardia Act, qui se rattache encore à une ère proprement libérale: "Norris-La Guardia was a laissezfaire approach to industrial relations, a strongly worded series of instructions to the federal courts to keep their hands off labour disputes because they had made a mess of things by intervening in the past"' (p. 27).

L'étude du système mis en place par le National Labor Relations Act de 1935 porte principalement sur la désignation des représentants collectifs, l'objet des différentes pratiques déloyales en matière de représentation et de négociation collective, la licéité du recours à différents moyens de pression surtout dans le cours d'une négociation collective, de même que sur le rôle privilégié de l'arbitrage prévu dans la convention collective. Dans ces pages, l'auteur décrit fort utilement les modes de fonctionnement du National Labor Relations Board et fait état également des principales prises de position des hautes juridictions fédérales. Le droit devient prétorien, comme lorsqu'il s'agit de jauger les comportements dans le cours de la négociation collective, ou encore l'objet obligatoire ou facultatif de cette dernière, le droit d'accès des représentants syndicaux aux lieux du travail, les limites du "free speech" des protagonistes, ou encore celles des pressions exercées sur des tiers ... L'auteur s'interroge aussi sur certains aspects de l'efficacité de fonctionnement du Board et des tribunaux d'arbitrage. Enfin, l'étude du droit des rapports collectifs se complète par celle du sort du salarié, dans ce contexte primauté de l'intérêt du groupe: les balises du devoir de représentation équitable, qu incombe aux représentants collectifs, et aussi, les devoirs du syndicat envers ses membres, selon le Landrum-Griffin Act. On présente finalement l'essentiel de la problèmatique du recours à la grève dans les services publics.

Le dernier chapitre s'intitule: "PublicInterest Labor Law". Il s'agit d'un résumé du Employee Retirement Income Security Act et du Occupational Safety and Health Act de 1970, ainsi que de l'importante législation contre la discrimination dans l'emploi. Pour l'auteur, ces sujets représentent "the new frontier of labour law" (p. 194). Certes, jusqu'à présent, le droit du travail américain misant essentiellement sur la négociation collective, aussi dynamique que conflictuelle, a 
su assurer la promotion sociale et économique d'une importante minorité de la main-d'oeuvre américaine. Pour la fin du siècle, de nouveaux défis, plus englobants, apparaissent en conclusion: la nécessité de formes de concertation dépassant l'entreprise, pour assurer une plus grande productivité et l'avènement d'une politique de revenus, de manière à contrer l'inflation et la concurrence internationale. Et puis, la répression légale de la discrimination dans l'emploi n'indiqueraitt-elle pas la voie plus large du contrôle du pouvoir de licenciement de l'employeur, dans les entreprises non syndiquées aussi bien que syndiquées? (p. 195)

La familiarité, voire le recul de l'auteur par rapport à cet ensemble relativement sophistiqué que représente le droit du travail américain de la négociation collective, lui permettent d'en livrer aussi simplement que valablement le contenu aux divers auditoires visés - représentants de la direction ou du personnel, juristes américains désireux de se familiariser davantage avec un système dont ils ne sont pas spécialistes, praticiens américains ou spécialistes étrangers animés d'un souci comparatiste ... Il lui est du même coup possible de proposer la direction probable de son évolution graduelle dans un sens plus généralement protecteur de l'ensemble des salariés.

\section{Pierre VERGE}

Université Laval

The Social Organization of Industrial Conflict: Control and Resistance in the Workplace, by P.K. Edwards and Hugh Scullion, Oxford, Basil Blackwell, 1982, 314 pp., ISBN 0-631-13127-2

Ce volume présente les résultats d'une recherche empirique sur le conflit industriel réalisée par deux chercheurs de l'Industrial Relations Research Unit, de l'University of Warwick. Paul Edwards et Hugh Scullion ont effectué une observation systématique dans sept usines en Grande-Bretagne, dont quatre de l'industrie mécanique, deux du secteur du vêtement et une autre produisant des fibres synthétiques.

L'orientation de la recherche vers le lieu même du travail, aux niveaux de l'atelier et de l'établissement, est probablement ce qui a le plus caractérisé les travaux du centre de recherche de Warwick au cours des dix dernières années. L'observation systématique, la réalisation de différents types d'entrevues et l'étude de sources documentaires (notamment les archives syndicales et d'entreprises) ont favorisé l'élargissement des connaissances sur différents aspects des relations du travail. Pensons aux travaux portant sur la détermination des salaires, l'organisation des délégués d'atelier, la réglementation par la négociation informelle et la coutume au niveau de l'atelier, ainsi que l'organisation et la médiation du conflit dans l'entreprise syndiquée. Sur ce dernier thème, le second volume de Batstone, Boraston et Frenkel, The Social Organization of Strikes, centrait l'attention sur le processus de mobilisation de l'action collective conduisant à la grève et à l'application d'autres moyens de pression. L'observation très systématique de l'action de certains délégués d'atelier faisait aussi ressortir leur rôle stratégique dans la médiation du conflit.

Par rapport aux recherches antérieures, on pouvait s'attendre à ce que l'observation sur les lieux de travail nous apprenne davantage sur l'origine et les sources du conflit, de même que sur les multiples formes de sa manifestation, au moment même de la production. Edwards et Scullion se sont penchés sur ces questions en plaçant le contrôle sur le procès de travail au centre de leur problématique.

Développant leur cadre d'analyse autour des concepts de contrôle sur le procès de travail et de résistance ouvrière, les auteurs mettent à profit les contributions d'auteurs marxistes tels Braverman, A.L. Friedman, R.C. Edwards et Burawoy. Cette contribution porte d'abord sur l'identification des fondements structurels du conflit. Ainsi, 'what is needed is a means of moving from the truism that conflit is inevitable to a way of identify- 